\title{
Individuación colectiva y emergencia de la organismalidad
}

\author{
Collective individuation and emergence of organismality
}

\author{
Isaac Hernández*; Davide Vecchi** \\ *Laboratoire de recherche ERRAPHIS, Département de philosophie, Université Toulouse Jean \\ Jaurès, France \\ hernandez.isaac@gmail.com \\ ${ }^{* *}$ Centro de Filosofia das Ciências, Departamento de História e Filosofia das Ciências, Faculdade \\ de Ciências, Universidade de Lisboa, 1749-016 Lisboa, Portugal \\ davide.s.vecchi@gmail.com
}

\begin{abstract}
Resumen
En este artículo nos centramos en la emergencia de la individualidad biológica por asociación, tratando de formular algunas condiciones teóricas para pensar el proceso de individualización colectiva. El punto de partida de nuestro análisis es el concepto de "transición evolutiva mayor". Una transición evolutiva mayor es el resultado de la integración de un cierto número de entidades biológicas, inicialmente independientes, que logran organizar sus interacciones, convirtiéndose en un colectivo de componentes que tiene una identidad orientada hacia un objetivo común. Cuando se trata de organismos, una transición mayor corresponde a un fenómeno de fusión entre organismos que, a veces, pertenecen a linajes diferentes. Así, el surgimiento de un nuevo nivel de individualidad biológica implica el establecimiento de relaciones constitutivas entre los individuos que cambia su estatuto de entidades autónomas. Como resultado, la aparición de un nuevo tipo de entidad en el mundo viviente implica que los individuos entablen relaciones que los transforman intrínsecamente, una transformación suficiente para que un "todo" se convierta en una "parte" que forma otro "todo", es decir; un nuevo nivel de organismalidad.
\end{abstract}

Palabras clave: individuación biológica, asociación, emergencia, organismalidad, complejidad biológica.

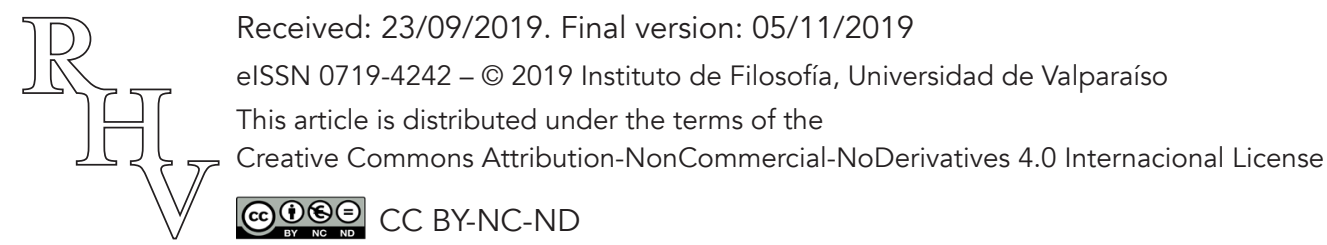


Individuación colectiva y emergencia de la organismalidad

Isaac Hernández; Davide Vecchi

\begin{abstract}
In this article we focus on the emergence of biological individuality by association, trying to formulate some theoretical conditions to think about the process of collective individualization. The starting point of our analysis is the notion of "major evolutionary transition." A major evolutionary transition is the result of the integration of a multiplicity of initially independent biological entities that, by managing to organize their interactions, become a collective of components having an identity oriented towards a common goal. When biological organisms (sometimes belonging to different lineages) are concerned, a major transition corresponds to a phenomenon of fusion between them. We shall argue that the emergence of a new biological level of individuality implies the establishment of constitutive relationships between individuals that change their status as autonomous entities. As a result, the emergence of a new type of entity in the living world implies that individuals enter into relationships that intrinsically transform them, a transformation sufficient for a "whole" to become a "part" that forms another "whole", that is, a new level of organismality.
\end{abstract}

Keywords: biological individuation, association, emergence, organismality, biological complexity.

\title{
1. Introducción
}

Todos los sistemas vivos poseen una dimensión interactiva que es indispensable para su auto-mantenimiento y existencia en un medio ambiente preciso. El conjunto de estas interacciones involucra la coexistencia con otros organismos. De hecho, desde el comienzo de la vida en la tierra, los organismos se han reagrupado o asociado, constituyendo grupos con distintos niveles de agregación y organización que aumentan, bajo ciertas circunstancias, las posibilidades de sobrevivencia de esos organismos (Ruiz-Mirazo y Moreno 2012). De esta forma, los organismos siempre han formado asociaciones de diferentes tipos, que poseen variados niveles de integración u organización que llevan a la creación de alianzas de distinto orden.

Ciertamente, podemos mencionar una variedad de colonias bacterianas, cuya dinámica opera bajo distintos niveles de complejidad que van desde agregaciones simples hasta agregaciones que poseen un gran nivel de complementariedad y coordinación de funciones. Tales ejemplos pueden referir a:

communities such as biofilms (which may be single or multi-taxa), as well as some populations of unicellular organisms, exhibit well-defined cell organization and a functional division of labour that includes specialized cell-to-cell interactions, the suppression of cellular autonomy and competition, metabolic collaboration, combined defense and attack strategies, and the coordination of movement, growth and reproduction. (Dupré y O'Ma1ley 2009, p.11)

Revista de Humanidades de Valparaíso, 2019, No 14, 335-362

(c) $(1)(9)$ CC BY-NC-ND 
Podemos referirnos también a las macro-interacciones ecológicas, en donde encontramos intercambios funcionales entre los organismos que comparten los recursos disponibles de un nicho común. De esta forma, podemos decir que la interacción entre organismos puede crear nuevos niveles de organización biológica, a partir del momento en que estas asociaciones logran anclar un nivel de integración importante.

Sin embargo, aunque existe un sinnúmero de ejemplos de comportamientos o fenómenos de asociación en el mundo vivo, los ejemplos de fenómenos de asociación que ven nacer un nuevo tipo de individuo biológico son más escasos. Podemos decir que una de las características más notables de este tipo particular de asociación es que las partes (organismos) que constituyen la organización actúan de manera coordinada, pudiendo eventualmente movilizar un proceso de selección natural al nivel de la organización. En efecto, la emergencia de un individuo biológico implica la existencia de un ritmo coordinado de actividad entre sus partes, una fenomenología única compuesta de entidades que fueron, en algún momento de la historia natural, entidades autónomas. De esta forma, comprender este fenómeno de asociación radica en el hecho de comprender cómo un grupo de individuos autónomos llega a hacer emerger un solo individuo autónomo.

En este artículo pretendemos profundizar conceptualmente en este fenómeno de integración particular, enfocándonos más específicamente en el problema de la emergencia de la individualidad biológica, a fin de comprender si y cómo un tipo particular de agregación de individuos pueden formar un solo individuo biológico.

\section{Transición evolutiva mayor}

Maynard Smith y Eörs Szathmáry en su libro The Major Transitions in Evolution introducen la idea de "transición" para denotar un cambio importante en la historia natural que no solamente puede alterar los tipos de entidades biológicas existentes, sino que también transforma el proceso evolutivo en sí mismo, abriendo así las puertas a la emergencia de nuevas formas de organización biológica, es decir, de complejidad biológica ${ }^{1}$.

En grandes rasgos, los autores sostienen que la evolución de la complejidad se ha canalizado, en varios momentos de la historia natural, a través de un puñado de grandes transiciones en los cuales encontramos grandes cambios en la manera en que la información se transmite de generación en generación. Así,

the evolution of new levels of biological organization, such as chromosomes, cells, multicelled organisms, and complex social groups radically changed the kinds of individuals

\footnotetext{
${ }^{1}$ Es importante precisar que las primeras tres transiciones postuladas por Maynard Smith y Szathmary (2010), no corresponden a asociaciones entre organismos, sino que a la evolución bioquímica de la biosfera. En este artículo, nos enfocamos en la evolución de nuevas formas de organismos por asociación y en la evolución de la organismalidad (Pepper y Herron 2008; Queller y Strassmann 2009).
}

Revista de Humanidades de Valparaíso, 2019, No 14, 335-362 
natural selection could act upon. Many of these events also produced revolutionary changes in the process of inheritance, by expanding the range and fidelity of transmission, establishing new inheritance channels, and developing more open-ended sources of variation. (Calcott et al. 2011, p1)

La evolución de la complejidad, al menos en este puñado de casos, sería una consecuencia de este particular fenómeno de transición, en donde la emergencia de un nuevo nivel de organización biológica implica la emergencia de novedad cualitativa.

Antes de continuar, es importante preguntarse por qué es necesario hablar de una transformación del proceso evolutivo. El hecho es que la teoría darwiniana de la evolución explica la variación de los organismos mediante un proceso de mutación aleatoria que se transmite de padres a hijos. La metáfora del árbol de la vida de Darwin pretende ilustrar las relaciones de parentesco entre grupos de seres vivos, teniendo cuidado en no considerar ningún tipo de proceso horizontal; ¡no se salta de una rama del árbol a otra! De esta manera, la novedad cualitativa que aparece a través del tiempo se explica únicamente en el espacio de la filogénesis de un clado siempre transmitida por descendencia directa. Podemos entender entonces por qué una transición mayor, en el sentido de Maynard Smith y Szathmary, podría cambiar nuestra visión del proceso evolutivo: de acuerdo con la visión darwiniana, el origen de los organismos que forman la especie proviene de la diferenciación de un organismo anterior que pertenece a la misma rama del árbol o al mismo linaje histórico, y no a un proceso de fusión entre organismos de diferentes orígenes. Sin embargo, en la idea de una transición mayor, la variación cualitativa puede ser impulsada también por fenómenos particulares de asociación entre individuos de diversos orígenes, cuando se asocian a tal punto que logran hacer emerger un nuevo nivel de individualidad. Así, la noción de transición mayor intenta capturar un fenómeno más general de evolución de la complejidad que podría tomar un camino diferente al de la evolución darwiniana clásica por descendencia común ${ }^{2}$.

El punto de partida de Maynard Smith y Eörs Szathmary para desarrollar la idea de transición evolutiva mayor es el reconocimiento de tres características principales de este fenómeno:

1. Las entidades que inicialmente fueron capaces de replicación autónoma pierden, después de la transición, esta autonomía replicativa en beneficio de una replicación dependiente de un conjunto más amplio.

\footnotetext{
${ }^{2}$ Se debe al mismo tiempo subrayar que Maynard Smith y Szathmáry (2009, p. 107) defienden la "excepcionalidad" de las fusiones por simbiogénesis: "The motorbike is a symbiosis between the bicycle and the internal combustion engine. It works fine, if you like that kind of thing, but someone had to invent the bicycle and internal combustion." El punto aquí es que la selección natural es más importante para entender la emergencia de las novedades evolutivas, y en consecuencia las transiciones mayores, que la simbiogénesis.
} 
2. Una transición mayor a menudo se asocia con la división del trabajo y la especialización de funciones.

3. Una transición mayor a menudo implica un cambio en la forma en que la información se transmite entre generaciones (Maynard Smith y Szathmáry 2010).

En este sentido, la aparición de la célula eucariota es un ejemplo paradigmático de este fenómeno. Hoy en día, el origen simbiogenético de la célula eucariota se impone frente a las otras teorías sobre la aparición de los eucariontes (Taylor 1976). Las mitocondrias y los cloroplastos (orgánulos semiautónomos de la célula eucariota) se definen como tipos de procariotas que han evolucionado especializaciones funcionales que les permiten generar y almacenar energía. Estas especializaciones se distribuyen, transmiten y heredan, inicialmente a través de la endo-simbiosis, luego como una función constitutiva de un nuevo organismo más complejo. En este punto, Lynn Margulis, una de las autoras de la teoría simbiogenética, especifica que los procesos de asociaciones que dieron lugar a la aparición de nuevas formas de vida a partir de la simbiosis (o en general a las sinergias entre las formas de vida existentes) son un motor fundamental en la transformación de la vida, en particular de su complejidad. Así, "long-term stable symbiosis that leads to evolutionary change is called 'symbiogenesis'. These mergers, long-term biological fusions beginning as symbiosis, are the engine of species evolution" (Margulis y Sagan 2002, 12). Como resultado, la teoría simbiogenética sostiene que las relaciones simbióticas que impulsan los procesos simbiogenéticos son responsables de gran parte de las novedades evolutivas, lo que puede ser consistente con la noción de transición evolutiva mayor, suponiendo que en cada transición hay un proceso de integración endógeno entre componentes de diferentes orígenes.

La idea de Margulis es en nuestra opinión fundamental para estructurar la noción de transición mayor. Un fenómeno de este tipo implica un flujo de fusión de individuos que, siendo profundamente afectados por los resultados de sus asociaciones, pierden su autonomía individual frente a un nuevo dominio de organización más complejo. De esta forma, una transición mayor podría definirse como el resultado de la integración de un grupo de organismos, inicialmente independientes, que hacen emerger un sistema unitario capaz de organizar sus interacciones como un colectivo de componentes que tiene una identidad orientada hacia un objetivo común. Es así como el resultado de cada evento de transición mayor es la aparición de un nuevo nivel de organización biológica con un nuevo nivel de complejidad. Es así también que el surgimiento de una nueva organización biológica autónoma implica la apertura de nuevos caminos de complejidad en los que las entidades emergentes son cualitativamente diferentes de sus predecesores. 


\subsection{Transición, complejidad y jerarquización}

Para entender mejor la relación entre la emergencia de la novedad cualitativa y el fenómeno de agregación de organismos asociado a una transición mayor, es importante entender el tipo de noción de complejidad que está en juego. En el caso de una transición mayor, esta complejidad se teoriza poniendo énfasis en los fenómenos de interacciones entre organismos, dentro de los cuales un conjunto de organismos logra estabilizar sus relaciones dando origen a un nuevo individuo biológico. Es importante entonces explicitar las conexiones teóricas entre el concepto de "nivel" en biología y la noción de "jerarquía", siendo estas nociones capitales para entender el proceso de emergencia de la complejidad que denotan las transiciones mayores.

Como decíamos, la complejidad está vinculada a la noción de "transición" y este vínculo se expresa a través de una jerarquización creciente en la historia de los seres vivos. En efecto, es común observar que los sistemas vivos tienen una estructura anidada o jerárquica que ha emergido a lo largo de la historia evolutiva. En términos generales, una jerarquía corresponde a una clasificación en la que los términos clasificados están en una relación de subordinación. De esta forma, una jerarquía expresa un tipo de relación de orden en el que hay elementos restringidos por otros elementos. En una organización, por ejemplo, la jerarquía se basa en un orden de prioridad entre los elementos o en las relaciones de subordinación entre los miembros de la organización ${ }^{3}$. Cuando decimos que la vida se nos aparece bajo una estructura jerárquica, decimos entonces que la vida tiene un tipo de arreglo u organización en el que los elementos están en una relación de subordinación. Cuando pensamos en esta relación desde la historia evolutiva, podemos decir que la evolución ha tendido a producir sistemas más complejos agregando progresivamente más niveles jerárquicos. Por ejemplo, si partimos desde las moléculas, estas moléculas se transforman posteriormente en biomoléculas, células, organismos multicelulares y grupos de organismos. Estas distintas etapas, caracterizadas por la aparición de un nivel mayor de complejidad, se pueden representar en una especie de escala en la que el elemento anterior se incluye dentro del nivel posterior.

Decíamos sin embargo que una jerarquía implica una subordinación de unos elementos respecto a otros. Una jerarquía entonces debe poder constreñir los niveles inferiores. Esto nos permite hacer una distinción teórica entre "jerarquía" y "nivel"4. En la idea de una

\footnotetext{
${ }^{3}$ Por ejemplo, en el ejército encontramos una disposición jerárquica de elementos, los soldados, donde el orden de prioridad de los elementos, los grados, es constitutivo de la organización.

${ }^{4}$ Esto es importante aclararlo para no confundir una jerarquía con un nivel de organización. Si el criterio utilizado para definir una jerarquía se basa únicamente en la inclusión de un nivel en el otro, en el que el nivel superior contiene entidades de nivel inferior al incluirlas como partes, el número de niveles jerárquicos existentes puede aumentar considerablemente. De hecho, un criterio como este, que puede considerarse a diferentes escalas, ya sea a nivel de los átomos hasta la biosfera, puede fácilmente volverse demasiado extenso, incluso ilimitado.
}

Revista de Humanidades de Valparaíso, 2019, No 14, 335-362 
transición mayor, dado que en cada transición surge un nivel de organización más complejo, podemos reconocer un número limitado de transiciones mayores $\mathrm{y}$, por lo tanto, de jerarquías bajo constricción. Por ejemplo, uno de los criterios de Maynard Smith y Szathmáry para definir una transición mayor es el hecho de que la entidad inferior pierde su autonomía replicativa o reproductiva durante la transición. Esto se ejemplifica en el caso de la transición de un nivel unicelular a un nivel multicelular, dado que el nivel superior restringe al nivel inferior a través de la pérdida de control de la función reproductiva del nivel inferior. Esta dependencia replicativa o reproductiva implica que el nivel superior constriñe el nivel inferior, lo que quiere decir que el nivel inferior está funcionalmente subordinado al nivel superior. En consecuencia, podemos decir que una transición mayor es la manifestación de un proceso de jerarquización en el que el número de niveles contenidos (o anidados) en la organización coexiste en una especificación jerárquica causal (McShea Daniel W. 2017), lo que Salthe llama "hierarchical process complexity" (Salthe, 1993). Por un lado, la jerarquización biológica obedece a una lógica de complejidad estructural (Heylighen 1999) en la que un sistema se compone de subsistemas acumulados, y las partes de un sistema estructuran el siguiente nivel, lo que también puede describirse como un aumento cuantitativo en los niveles organizacionales a lo largo del tiempo evolutivo. Por otro lado, en una transición mayor existe también una complejidad cualitativa que se constata al analizar los cambios y novedades biológicas, como cuando el sistema sufre un cambio fundamental en la forma en que almacena y transmite la información hereditaria (Szathmáry 2015).

Así, podemos decir que, en un proceso de individualización colectiva, existe un fenómeno de constricción en donde el nivel emergente se define por la constricción sistemática de sus elementos. De esta manera, la formación de un nuevo nivel de complejidad, en el sentido de una transición mayor, coincide con la formación de un nuevo nivel jerárquico. Así, la complejidad creciente de la vida se produce bajo un número limitado de transiciones evolutivas mayores, cuya particularidad es que el nuevo dominio relacional (el nivel superior) ejerce una determinación sobre los elementos que lo componen ${ }^{5}$. Como veremos más adelante, es también en este sentido que Maynard Smith y Eörs Szathmáry hablan de complejización durante la evolución, sugiriendo, entre otras condiciones, la pérdida de funciones de los individuos que componen la nueva organización.

Podemos resumir conceptualmente lo anterior diciendo que una transición evolutiva implica una fusión de individuos que permite la aparición de un nuevo tipo de individuo biológico. Este individuo representa un dominio biológico de organización distinto al de los individuos que se fusionaron. La aparición y persistencia de este nuevo dominio de

\footnotetext{
${ }^{5}$ Como podemos ver, existen distintas caracterizaciones de la noción de complejidad. Nosotros hemos hablado de complejidad jerárquica, cuantitativa y cualitativa en conexión con el tema de la emergencia de novedades biológicas. Lo importante es entender que todas estas nociones se encuentran imbricadas en una transición mayor.
} 
organización biológica depende, sin embargo, de la subordinación de las partes al nuevo nivel o dominio organizacional. Por lo tanto, si los individuos que componen este dominio están necesariamente subordinados para la realización de sus ontogénesis a las restricciones relativas de la organización del nivel superior del cual forman parte, entonces hay una nueva unidad, o individuo, que emerge ${ }^{6}$. Si este nuevo nivel de organización se estabiliza, el sistema puede ser seleccionado como un individuo en el que la evolución de las unidades componentes está necesariamente subordinada a la evolución de la nueva unidad compuesta. Finalmente, la estabilidad de este nuevo dominio biológico producido por la fusión entre individuos de orígenes a veces diferentes, se da cuando la fenomenología de los componentes está orientada hacia el mantenimiento de la organización de la unidad global, siendo esta una condición del mantenimiento y persistencia de la organización de los componentes.

\subsection{La composición de una jerarquía}

Para articular la noción de jerarquía con la noción de transición evolutiva, podemos centrarnos en la acumulación de los niveles de organización presentes en las jerarquías más complejas, o recientes, de la vida. Esto conecta también con la idea de tendencia a la complejidad creciente observada a lo largo de la historia de la vida ${ }^{7}$, en el sentido que la inclusión de un nivel en otro implica que este último es más complejo que el primero, en términos de los niveles incorporados en su estructura. Al mismo tiempo, una tendencia a la complejidad en la vida, de manera abstracta, también implica una tendencia a la jerarquización, es decir, una tendencia en "structural hierarchy, or the number of levels of nestedness of lower-level entities within higher-level individuals, of parts within wholes" (McShea 2001, p. 405). Así, la noción de jerarquía y de complejidad se encuentran en una relación directa $^{8}$.

\footnotetext{
${ }^{6}$ Es de precisar que una subordinación causal implica la aparición de una nueva norma biológica: todos los individuos que pertenecen a la organización colectiva dependen, para existir, de la organización global. Con el término "norma" se entiende una dinámica de asociación estabilizada. Por ejemplo, la normatividad fisiológica se manifiesta cuando las funciones de la asociación dependen las unas de las otras. En el sentido de Kaneko et al. se asocia la norma a una sociedad organizada en donde cada elemento se armoniza en conjunto y coexiste; en este caso Kaneko trata más bien la emergencia de la norma como orden colectivo (Furusawa y Kaneko 1998b).

${ }^{7}$ Justificar teóricamente que la vida tiene una tendencia a la complejidad es polémico. Solo podemos decir que hasta ahora la vida ha mostrado una tendencia a la complejidad lo suficientemente obvia como para movilizar varias hipótesis que intentan explicar esta tendencia (Bedau 2009).

${ }^{8}$ Si bien la existencia de una tendencia creciente a la complejidad por acumulación permanece controvertida en la literatura, sobre todo relativamente a la existencia de tendencias muy fuertes a la reducción genómica (O’Malley et al. 2016; Wolf y Koonin 2013). Se ha argumentado que, a pesar de la tendencia a la simplificación de la morfología, la tendencia a la complejización permanece creciente. La idea detrás de esta última posición es que la tendencia a la complejidad no siempre ocurre a través de la «acumulación» de
} 
Si nos centramos en un criterio estructural de la complejidad, podemos establecer un criterio teórico y operativo para comprender la misma. Los aspectos estructurales de la jerarquía consisten en especificar de cuántos tipos de entidades se compone el nivel jerárquico, así "by this criterion, a multicellular eukaryote contains more levels than a solitary prokaryotic cell; a society of multicellulars, more levels than a solitary multicellular individual" (McShea y Brandon 2010, 47). Es así como Daniel McShea propone un criterio cuantitativo para abordar la complejidad, basado en una descomposición del número de niveles entrelazados de la estructura jerárquica y el grado de individualización de la entidad de nivel más alto. Esto supone un principio de inclusión, en donde la entidad de nivel más alto incluye el nivel inferior como sus partes. Materialmente hablando, el nivel superior está compuesto de las partes del nivel inferior. Al mismo tiempo, para evitar la posibilidad de establecer jerarquías biológicas de forma ilimitada, es necesario agregar la condición siguiente: "lower-level entities [...] must be homologous with organisms in a free-living state, either extant or extinct" (McShea 2001, p. 408). Esto permite establecer un criterio más preciso en la definición de jerarquía biológica que limita el número de niveles jerárquicos que corresponden a las transiciones evolutivas. Así, por ejemplo, si la célula procariota ocupa el primer nivel de análisis jerárquico, el segundo nivel correspondería a la célula eucariota, dado que esta célula es una composición de procariontes. El siguiente nivel jerárquico sería los multicelulares, siendo estos una composición de eucariontes, así como los organismos coloniales serían una composición de los multicelulares. En todos estos casos, cada nivel jerárquico está compuesto del anterior, cuyos elementos son homólogos, en estado de libertad y autonomía, con el nivel superior que componen. A partir de este criterio, un órgano del organismo (el corazón, por ejemplo) no es homólogo a un organismo en estado de libertad, precisamente porque un órgano no puede concebirse por separado del organismo, es decir como organismo autónomo y libre ${ }^{9}$ La mayoría de las caracterizaciones de la jerarquía biológica se basan implícitamente en este requisito de existencia en autonomía y libertad; sin ellas, no habría límite teórico sobre la profundidad jerárquica discernible en los sistemas biológicos (Pigliucci 2010).

En esta sección, hemos querido establecer un sustrato conceptual para pensar en las condiciones para la emergencia de un nivel de individualidad. Al respecto, es importante enfatizar que la especificación apropiada de un nivel jerárquico se justifica cuando nos

partes y tipos de partes (por ejemplo, por diversificación funcional), sino que, a veces, a través de la perdida de ellas. Nótese también que esta posición es coherente con la idea extrapolada de la mereología formal; cuando el todo se vuelve más complejo, las partes se vuelven más simples (Varzi 2016).

${ }^{9}$ Esto parece ser un ejemplo similar al de las mitocondrias o el cloroplasto, que se han convertido en órganos especializados en el metabolismo de la energía celular. Incluso si en este caso reconocemos una cierta autonomía, sus orgánulos ya no pueden sobrevivir fuera de la maquinaria celular. En efecto, usando el criterio de homología de McShea, la mitocondria o el cloroplasto, siendo homólogos a organismos libres del pasado, no son análogos al corazón: el hecho de llamar a la mitocondria y el cloroplasto «organelos» no significa que son análogos a órganos en este sentido (McShea y Hordijk 2013).

Revista de Humanidades de Valparaíso, 2019, No 14, 335-362 
encontramos, como veremos a continuación, con el problema de especificar los niveles pertinentes para pensar en la individualidad biológica que supone la selección darwiniana. En este caso, los niveles relevantes de individualidad biológica se limitan a los niveles análogos a los organismos autónomos, evitando así estructuras y niveles intermedios. Así, contrariamente a una visión de la individualidad biológica en la que cada nivel de organización puede concebirse teóricamente como un nivel de selección, se puede sostener que los niveles relevantes para hablar de individualidad corresponden únicamente a los niveles expresados en las transiciones evolutivas. Es decir, en las que un conjunto de organismos ha logrado estructurarse bajo un espacio de interacción estructural/organizacional, de manera que sus comportamientos están correlacionados. Es así que la disposición de un conjunto de individuos que han establecido relaciones constitutivas con sus pares resulta en una agrupación que toma la forma de un nuevo dominio relacional que difiere de otros dominios relacionales del mundo exterior.

A su vez, hemos insistido que, en el marco de una transición evolutiva mayor, los organismos pierden gradualmente su autonomía fisiológica y reproductiva en provecho de una organización mayor de la cual ellos forman parte. El punto central es que las partes "renuncian" a ciertas funciones que permiten su autonomía. Como veremos a continuación, nuestra interpretación es que, en un proceso de individuación colectivo, debe haber una transferencia de autonomía por parte de los individuos que se ajustan al nuevo dominio colectivo.

\section{De una multiplicidad a una unidad: la transición}

El enfoque teórico del programa de investigación de "transiciones mayores" se centra en los episodios de creación de los nuevos tipos de agentes evolutivos, investigando cómo la individualidad biológica se transforma a través de la historia natural. En esta sección, mostraremos cómo es posible concebir, desde un punto de vista evolutivo, la aparición de un agente evolutivo.

\subsection{La composición del individuo biológico darwiniano}

El tema de la individualidad biológica se ha convertido en una unidad teórica fundamental desde el desarrollo de la teoría de la evolución por selección natural. De hecho, la explicación evolutiva requiere individuos, a menudo en competencia, que constituyan poblaciones en evolución. La explicación evolutiva también introduce una perspectiva diacrónica. Los individuos son concebidos como productos de la evolución, y estos pueden, al parecer, identificarse en muchos niveles de la jerarquía biológica (Lewontin 1970). David Hull define el individuo biológico como "spatiotemporally localized cohesive and continuous entities (historical entities)" (Hull 1978, p.336). El aspecto histórico de esta definición nos coloca directamente en el tiempo evolutivo. En este tiempo, el proceso de

Revista de Humanidades de Valparaíso, 2019, No 14, 335-362

(c) $(9)(9)$ CC BY-NC-ND 
selección natural representa la fuerza motriz de la evolución, la misma fuerza que Darwin anunció en términos de la "lucha por la existencia" de los individuos en un entorno de recursos limitados. Desde la teoría de la evolución por selección natural, y especialmente a través de su formulación contemporánea conocida como "la nueva síntesis" (Mayr 1998), el enfoque evolutivo de la individualidad biológica es dominante en las discusiones teóricas de la individualidad biológica. Así, la cuestión del individuo biológico ha sido absorbida por la noción de "unidad de selección" (Hull 1980; Godfrey-Smith 2009; Clarke 2010, Wilson 2005; Ghiselin 1971). Esta es la noción más ampliamente utilizada para definir un individuo biológico, subrayando su carácter evolutivo (Clarke 2010). Un individuo biológico corresponde, según esta noción, a una entidad sujeta a la fuerza de la selección natural, cuyo poder causal actúa sobre esta entidad-unidad discreta y continua. En consecuencia, podemos decir que el criterio ampliamente utilizado sobre la individualidad biológica se basa en la posibilidad de ser un objeto de selección (Mayr 1997).

Sin embargo, si bien la teoría de la evolución darwiniana a menudo se ha interpretado poniendo énfasis más bien en la competencia que en los fenómenos de cooperación, parece difícil negar que muchos organismos, para competir más eficazmente con sus pares, establecen nichos cooperativos. Basta pensar en el mutualismo establecido entre la célula huésped y las mitocondrias/cloroplastos que forman las células eucariotas, o al mutualismo de los biofilmes, o al mutualismo de los holobiontes, o a la co-evolución entre las plantas y sus polinizadores. En efecto, los ecosistemas no son sólo lugares de competencia, sino también de redes de interdependencia y de comunidades de las cuales depende la integridad de todos los miembros (Leigh and Vermeij 2002). Por lo tanto, si bien el criterio mayoritario para pensar la individualidad biológica se basa en la posibilidad de ser una unidad de selección, la cuestión de la emergencia de un nuevo individuo biológico, en el contexto de una transición mayor, se ha abordado a través de la siguiente pregunta: ¿cómo un grupo de individuos se vuelve un solo individuo, susceptible de desencadenar un proceso de selección al nivel de la colectividad, sin que la selección individual impida tal colectividad? (Michod and Nedelcu 2003a). De hecho, como señala Richard Michod, desde una perspectiva evolutiva, los individuos son "conjuntos" integrados e indivisibles que tienen un valor selectivo (fitness) que puede ser heredable; por lo tanto, pueden evolucionar y adaptarse a su propio nivel de organización (Michod 2007).

Para pensar en la evolución de grupos de organismos en grupos bien integrados y organizados, podemos suponer una organización robusta, un comportamiento social particular que conduzca a la estabilidad del grupo. Como explicaremos más adelante, esto significa que las relaciones entre los individuos en el grupo deben ir más allá de los beneficios individuales. El problema en cuestión es si la selección puede actuar a un nivel diferente al de los individuos, cuando estamos en presencia de tales comportamientos sociales. Si este es el caso, debe postularse que la selección actúa, no solo al nivel del individuo, sino que también puede actuar, bajo ciertas circunstancias, al nivel del grupo, lo que permitiría considerar una selección "multinivel” (Martens 2012; Okasha 2008). Por otro lado, la 
idea de que los grupos de organismos pueden hacer emerger propiedades que pertenecen exclusivamente al grupo y que son análogas a las de los individuos que conforman el grupo - lo que permitiría que estos grupos puedan ser concebidos como unidades de selección - nunca ha sido fácil de justificar. De hecho, la supuesta incompatibilidad de la selección con la cooperación ha provocado una serie de conflictos entre detractores y defensores de Darwin (Leigh 2010). Es Darwin en persona quién reconoce explícitamente en "El origen de las especies" que hay al menos un fenómeno biológico, los insectos sociales, que plantea "una dificultad muy especial lo suficientemente [...] insuperable como para derrocar" (Darwin 2008, p. 299) su teoría, sabiendo que en este tipo de sociedades la selección natural no parece ser aplicable a todos los individuos de la misma manera ${ }^{10}$. Sin embargo, incluso si Darwin postula, en el caso particular de las sociedades de insectos, la acción de una selección a nivel del conjunto, recordando que "la selección se aplica tanto a la familia como al individuo" (Darwin 2008, p. 301), la biología de la evolución ha dedicado mucho tiempo a teorizar la selección de los organismos paradigmáticos que pertenecen en particular al mundo-macro. Se piensa a menudo que la selección natural actúa ineludiblemente en organismos individuales, ya que la selección natural presupone, independientemente del nivel en cuestión, una replicación diferencial de entidades discretas. Por supuesto, esta es una condición que los grupos no satisfacen a primera vista. Es desde esta perspectiva que podemos entender la exclusión sistemática de un enfoque que piensa la selección a un nivel diferente al del organismo individual (Leigh 2010).

Para pensar una tal problemática, es interesante remitirse al origen simbiótico de la célula eucariota, que de hecho ha permitido modificar la representación que anteriormente se hacía de los mecanismos de evolución. El hecho es que este evento particular de la historia evolutiva pertenece a un proceso de fusión de linajes, y no al resultado de la variación de un linaje fisionado, como lo requiere la síntesis moderna. En efecto, la apertura al micro-mundo muestra claramente que la biología evolutiva, centrándose durante mucho tiempo en los procesos evolutivos de organismos individuales del macro-mundo, ignoró la importancia de los procesos horizontales. En particular, ha omitido la posibilidad de que los linajes puedan fusionarse para formar un sistema organizado completo que logre generar un nuevo individuo biológico (Doolittle y Bapteste 2007).

\subsection{Una correlación inversa de fuerzas}

Las interacciones entre individuos, en las cuales el surgimiento de un individuo evolutivo es uno de los resultados posibles, a menudo parecen motivadas por los beneficios que aporta su asociación, mostrando así características y propiedades adicionales, donde los intercambios han podido evolucionar desde la formación de grupos, hacia la formación, en el extremo, de verdaderos "super-organismos". De hecho, tan pronto como se evoca un

\footnotetext{
${ }^{10}$ Con toda certeza debido a la imposibilidad de propagación de los miembros estériles.
} 
proceso de individualización colectiva, examinamos la evolución de estos intercambios. Entre estos intercambios, la cooperación aparece como un elemento central de una organización colectiva que va más allá de los intercambios puramente individuales.

El surgimiento de la cooperación, bajo su faceta estratégica, la podemos entender como el "bien común". Sin embargo, tal bien común representa una complejización de las relaciones entre dos tipos de selección: la selección natural individual y la que tiene lugar al nivel del grupo en lo que esta última parece optimizada en comparación con los individuos aislados. De hecho, una población compuesta por individuos que dedican sus acciones a la preservación de sus estados individuales se sitúa es un proceso de selección individual donde la competencia se impone frente a la colaboración entre individuos. Por otro lado, tan pronto como tenemos una población que establece relaciones de interdependencia, nos movemos al otro lado del fenómeno de selección, con una fuerza selectiva que se desplaza hacia el lado del grupo, dejando espacio para la colaboración como vector de orden. Nos gustaría expresar esto por el término correlación negativa. Esto implica que la optimización de una colectividad pasa por una especie de inversión de la fuerza de selección individual, en donde el peso de la fuerza de selección es mayor a nivel de la comunidad que a nivel de los individuos que componen el grupo. Así, se da una correlación negativa entre la fuerza de selección del nivel de integración del colectivo y la fuerza de la selección individual de los elementos que componen el colectivo.

Este fenómeno de inversión entre la selección individual y la selección de grupos ha estado en el centro de la investigación sobre el comportamiento social, estableciendo la cooperación y su evolución como una forma de resolver el problema de la coexistencia de los varios niveles de organización biológica (Sober y Wilson 2003; Wilson 1997; Wilson y Sober 1989). Si pensamos en el problema de la compatibilidad de varios niveles de organización biológica, podemos suponer que el nivel inferior debe favorecer la cooperación a expensas de la competencia, lo que permite una disminución en la fuerza de selección individual y un aumento en la fuerza de selección a nivel de grupo. La evolución de la cooperación representa un paso crucial en el diseño de la transición efectiva de un estado individual a un estado de poblaciones estructuradas en grupos altamente organizados. Esta es la razón por la cual una transición evolutiva implica la formación de grupos cooperativos que se convierten, en ciertas circunstancias, en entidades integradas $\mathrm{y}$ vinculadas que pueden considerarse como nuevos niveles de individualidad.

Sin embargo, no es fácil teorizar la colaboración como condición para el surgimiento de una comunidad organizada. $\mathrm{Si}$, por un lado, la cooperación tiende a maximizar los beneficios selectivos de los grupos cuyos miembros actúan en coordinación (optimizando sus relaciones como grupo), por otro lado, la cooperación implica un costo para ciertos individuos que pertenecen a este grupo. Este costo puede atribuirse simplemente a su compromiso o al esfuerzo requerido durante la cooperación. Por ejemplo, el producir en exceso un elemento funcional para el grupo, proteger al grupo, prevenir posibles peligros, todos estos comportamientos benefician al conjunto, pero les cuestan a los cooperadores 
individuales sus inversiones energéticas en estas tareas. Así, la problemática de pensar en la cooperación como una fuerza impulsora de optimización de grupos hacia un estado de individualidad colectiva puede formularse de la siguiente manera: por un lado, la cooperación emerge como un proceso que reúne los intereses de todos, en el sentido en que un grupo que posee muchos colaboradores recolecta lógicamente un beneficio selectivo de cada individuo de ese grupo; por otro lado, el efecto lógico del costo de la colaboración recae en los miembros colaboradores y peligra el riesgo de propagación de la deserción. En este último caso, la selección natural debería causar una disminución de la frecuencia de estos cooperadores, lo que llevaría a una reducción de la viabilidad promedio de los grupos y, paradójicamente, a la pérdida de la posibilidad de generar un nuevo nivel estable de organización ${ }^{11}$.

Como podemos ver, este problema no es fácil de abordar. Esta problemática ha estado en el centro del debate sobre los estudios del altruismo biológico, ya que este rasgo es uno de los pilares fundamentales para pensar en la aparición de grupos sociales estables que creen un nuevo dominio biológico. En el contexto de una transición mayor, la mayoría de los niveles jerárquicos (genes, redes genéticas, células, células eucariotas, organismos multicelulares, super-organismos, etc.) evolucionaron a partir de un proceso de individualización colectivo que se caracteriza por un proceso de fusión entre individuos, ya que estos individuos se vuelven interdependientes. Esto se estudia en el enfoque de las transiciones mayores como resultado de la evolución de la cooperación. En consecuencia, si bien la evolución de la cooperación ha demostrado que el hecho de cooperar, incluso si a veces implica que ciertos individuos sacrifican sus beneficios individuales, hace posible favorecer al grupo en detrimento de los grupos menos colaboradores, es posible que la selección natural haya favorecido la permanencia de los rasgos altruistas en grupos cuyos beneficios selectivos promedio fueron mayores. De hecho, los grupos altruistas son más aptos, en el sentido de que cuentan con ventajas selectivas más importantes que los grupos no altruistas, lo que puede favorecer la evolución de este rasgo a pesar de las desventajas individuales en los grupos (Sober y Wilson 2003). Esto puede explicarse por el hecho de que la cooperación, aunque implica un cierto costo no despreciable dentro de los grupos, también tiene ventajas que explicarían por qué persisten estas asociaciones: en los grupos colaboradores, hay funciones que emergen que los miembros no pueden desarrollar por sí mismos. Por lo tanto, parece razonable decir que el nacimiento de la colaboración, a veces expresado como un sacrificio de autonomía individual, representa un paso crucial en el camino hacia la individualización de un grupo. Así, el tratamiento de la problemática de la cooperación requiere una comprensión del altruismo y su evolución dentro de los grupos, siendo esto la clave para entender cómo funciona la colaboración como principio de optimización.

\footnotetext{
${ }^{11}$ En la literatura este problema teórico es llamado "subversion from within" (Baron, 2013).
} 


\section{Condiciones teóricas para pensar la individuación biológica}

Como decíamos anteriormente, la consecuencia lógica de la cooperación dentro de los grupos radica en el hecho de que, para superar las dificultades teóricas planteadas por la selección individual en un contexto de individuación colectivo, necesitamos que la cooperación evolucione. Esto establece las condiciones para pensar en el surgimiento de una colectividad organizada: los individuos deben trabajar juntos con altos niveles de cooperación y bajos niveles de conflicto. Esta condición retoma la noción de organismo de Queller y Strassmann; "the organism is simply a unit with high cooperation and very low conflict among its parts" (Queller y Strassmann 2009). Esto significa que un proceso de individualización colectivo pasa por una adaptación en la que el conjunto ha logrado disminuir la competencia y aumentar la colaboración. Esto se traduce en el organismo a través de la supresión (idealmente) de la perturbación de las adaptaciones a niveles inferiores. En una transición mayor, encontramos mecanismos a través de los cuales un grupo de organismos se convierte en un solo organismo, es decir, un grupo que tiene un nivel muy bajo de conflicto y un nivel muy alto de cooperación entre sus miembros.

A continuación, expondremos algunas hipótesis de las condiciones para la formación de comunidades optimizadas y organizadas como agentes evolutivos. Estas condiciones están principalmente asociadas con la evolución de la cooperación y el control de conflicto entre los miembros.

\subsection{Especialización y control de conflictos}

Hemos dicho que una transición mayor se puede interpretar como un escenario en el que los individuos que antes eran autónomos se vuelven dependientes del nivel superior, es decir, de la red de interacciones que se han formado entre los individuos. Esta dependencia consiste en el hecho de que estos individuos se convierten en partes especializadas de un nuevo nivel de organización. Esta especialización se traduce en la conversión de los individuos previamente autónomos en elementos funcionales de un nuevo dominio biológico. Esto también puede entenderse como un proceso de transferencia de funciones al nivel superior, lo que no es otra cosa que la evolución de la especialización ${ }^{12}$. Es así que la idea de dependencia funcional observada durante una transición mayor encaja bien con la noción de indivisibilidad contenida en la noción de individuo. Cuando se produce una especialización de individuos de nivel inferior en funciones vitales (como la reproducción o funciones somáticas en organismos multicelulares), la organización general se vuelve, en última instancia, indivisible, es decir, se convierte en un verdadero individuo. Por lo tanto, la división del trabajo, resultante de la especialización de los individuos, también termina estableciendo una dependencia mutua: los individuos se vuelven tan especializados que pierden la capacidad de realizar otras tareas de manera autónoma (West et al.

\footnotetext{
${ }^{12}$ Este punto lo tratamos en la sección 4.2.
} 
2015). La especialización así es crucial en un proceso de individualización colectivo. En efecto, se pueden distinguir dos tipos de especializaciones: por un lado, está la división del trabajo fisiológico; por otra, la división del trabajo reproductivo. Cuando ambas se actualizan, se reúnen las condiciones para la emergencia de un nuevo individuo biológico. Si pensamos en la aparición de una unidad a partir de un conjunto heterogéneo de individuos, como es el caso de la aparición de la célula eucariota o de los primeros organismos multicelulares ${ }^{13}$, podemos comprender mejor la importancia de los dos tipos de especialización. La interdependencia funcional fisiológica, es decir, el hecho de que la función fisiológica de otro se vuelve indispensable para la estabilidad de uno y vice-versa, parece esencial para estabilizar (por ejemplo, para la emergencia de normas) un grupo que mantiene relaciones constitutivas. Si agregamos la interdependencia funcional reproductiva, podemos entender lo que Queller parece decirnos:

if independent units are to come together into successful new organismal entities, there must be some means of controlling the conflicts between them, some means of ensuring that the old units do not continue to pursue their individual reproduction at the expense of the group. (Queller 2000, 1650)

La división del trabajo fisiológico y reproductivo se enlaza con la emergencia de mecanismos de control de conflicto. Un ejemplo interesante de control de conflicto es el fenómeno del desarrollo embriológico. La aparición de mecanismos de control de conflicto ha podido evolucionar hacia mecanismos precisos de desarrollo observables en los complejos organismos actuales ${ }^{14}$. La aparición de mecanismos de desarrollo en el fenómeno de la multicelularidad puede entenderse como una consecuencia general de la interacción entre la dinámica interna y externa de las sociedades celulares (Furusawa y Kaneko 1998a; Kaneko y Furusawa 2000; Kaneko y Yomo 1999). En otros términos, puede entenderse como un mecanismo general de diferenciación espontánea de unidades biológicas que, al replicarse, se diferencian por interacción con otras células, desde el momento en que sus números aumentan por divisiones (Furusawa y Kaneko 1998a). Así, el origen de las organizaciones multicelulares puede describirse como una consecuencia posible de la dinámica interactiva de las células, sin que sea necesario postular un mecanismo genético o preformado que limite el comportamiento celular (Vecchi y Hernández 2014). La evolución de los mecanismos de control que supone una organización multicelular, corresponde originalmente a un fenómeno espontáneo y aleatorio de diferenciación funcional asociado con el comportamiento interactivo de las células. Estos mecanismos pueden haber evolucionado hacia la aparición de funciones del desarrollo embriológico

\footnotetext{
${ }^{13}$ Véase algunos interesantes aportes teóricos sobre el surgimiento de la multicelularidad en: Newman et al. (2006), Furusawa y Kaneko (2002).

${ }^{14}$ Sobre este punto, consultar las ideas de Newman y Bhat (2009).
}

Revista de Humanidades de Valparaíso, 2019, No 14, 335-362 
muy sofisticadas y complejas ${ }^{15}$. Este "orden", producido a partir de la interacción entre las células y su dinámica intracelular, conduce a la aparición de normas. Su producción ocurre espontáneamente en la aparición de funciones que ordenan poco a poco las interacciones celulares, alcanzando con el tiempo un proceso de desarrollo robusto que mantiene un grado de orden en una sociedad celular (Furusawa y Kaneko 1998b). Finalmente, en el caso de grupos multicelulares, el control del conflicto puede implicar la propagación de mediadores de conflictos que conducen al autocontrol, el control materno del destino celular, la disminución del tamaño de los propágulos, el crecimiento determinado del organismo, respuestas apoptóticas o el secuestro de la línea germinal (Michod y Nedelcu, 2003b).

Llegado a este punto, podemos pensar que la aparición de mecanismos de mediación de conflicto, en grupos colaboradores, corresponde a las primeras funciones que hacen posible convertir un grupo en una unidad. El surgimiento de la individualidad, por lo tanto, depende del surgimiento de funciones colectivas, incluidas las funciones que restringen el conflicto al garantizar la estabilidad y la armonía de las unidades de nivel inferior. Entonces, una de las diferencias cruciales entre un grupo de individuos y una unidad compuesta de individuos radica en el hecho de que el segundo posee, entre otras cosas, funciones que aseguran la persistencia de los miembros que componen el nuevo dominio biológico. En este sentido, decimos que el poseer mecanismos que conducen a la armonía multinive ${ }^{16}$ corresponde finalmente a una de las funciones de nivel superior que surgen durante el comportamiento relacional de la vida. Por lo tanto, la emergencia de propiedades colectivas, como la función del desarrollo, puede servir como un criterio de distinción entre los verdaderos sistemas individualizados, que ponen de manifiesto las funciones colectivas, y los agregados de individuos que no han establecido relaciones constitutivas y que, por lo tanto, no corresponden a individuos reales.

\subsection{Transferencias y distribución de funciones}

En la sección 3.2 señalamos que una correlación negativa entre las partes y el todo hace posible pensar en la forma en que se armonizan los niveles de organización que conforman una comunidad. Al mismo tiempo, esta correlación negativa se presenta como una condición necesaria para que un conjunto de individuos se estabilice como unidad. También explicamos que, detrás de esta correlación negativa, existen mecanismos o funciones colectivas que gestionan la competencia entre los miembros de un grupo, lo que permite

\footnotetext{
${ }^{15}$ Este tema se estudia en relación al comportamiento de los sistemas complejos, especialmente porque durante las interacciones celulares, la célula, como sistema complejo lejos del equilibrio termodinámico, describe condiciones iniciales que conducen a la aparición de un orden del caos. Véase: (Goodwin 2001; Newman y Bonner 2016; Solé y Goodwin 2000).

${ }^{16}$ Creemos que los mecanismos de desarrollo son un muy buen ejemplo de control de conflicto. Se trata de una función que permite manejar una multiplicidad de individuos en un solo dominio organizacional.
}

Revista de Humanidades de Valparaiso, 2019, No 14, 335-362 
una armonización del nivel inferior con el nivel superior. Esto se debe a que el control de la competencia y del conflicto hace posible dirigir la fuerza selectiva hacia el grupo; la comunidad puede desencadenar un proceso de selección natural a nivel de conjunto. Por lo tanto, un proceso de individualización colectiva depende del surgimiento de funciones de nivel superior que limitan la posibilidad de confrontaciones entre los miembros, asegurando que la cooperación continúe entre las unidades de nivel inferior. Controlar el conflicto conduce, a través de la evolución de las adaptaciones que lo reducen, a una mayor individualidad y una mayor armonía para el organismo (Michod y Roze 2001).

Para explicar lo anterior en términos más concretos, Leo Buss (Buss 1987) propone el proceso de secuestro temprano de la línea germinal como mediador del conflicto durante la transición de la multicelularidad. Esto se debe a que dicha adaptación corresponde a un tipo de especialización funcional que hace que los miembros del grupo sean interdependientes y, por lo tanto, el sistema que constituyen indivisible. En este sentido, el secuestro de la línea germinal, como un caso de adaptación específica de la multicelularidad, supone la división funcional reproductiva entre las células somáticas y germinales (Huneman 2013). Una consecuencia interesante de esta división funcional es la interdependencia funcional que presupone. Un sistema dividido en funciones imprescindibles, como la reproducción y las funciones somáticas (el metabolismo, por ejemplo), conduce a una dependencia funcional vital, en el sentido de que cada individuo (o tipo de célula) necesita la función del otro para existir. En otras palabras, una división funcional conduce a una dependencia constitutiva irreversible.

Al mismo tiempo, el problema central que surge cuando se investiga la aparición de un nuevo nivel biológico es entender cómo puede haber una conciliación de varios niveles de organización en los que cada nivel está sujeto a las fuerzas de la selección natural en su propia escala. Richard Michod sugiere la transferencia de fitness ${ }^{17}$ como un medio para conciliar los varios niveles de organización biológica. Michod apunta que el pasar de un estado de selección individual a un estado de selección colectivo puede llevarse a cabo sobre la base de una transferencia de fitness entre el nivel inferior (los organismos individuales que estructuran la colectividad) y el nivel superior (la colectividad que contiene los individuos cuya aptitud diferencial disminuye respecto a la aptitud diferencial de la colectividad). La fitness o "aptitud" de cualquier unidad evolutiva puede entenderse de acuerdo con dos componentes básicos: fertilidad (reproducción) y viabilidad (mantenimiento y

\footnotetext{
${ }^{17}$ La fitness o "aptitud" es una herramienta descriptiva útil para los estudios de selección natural y se asocia con la tasa de supervivencia y el éxito reproductivo de un individuo o población. Los diferentes genotipos/ fenotipos de una población nunca tienen las mismas capacidades reproductivas ni las mismas tasas de supervivencia. En consecuencia, los individuos mejor adaptados a un entorno tendrán una mejor oportunidad de reproducirse en ese entorno; producirán por esta razón más descendientes. La importancia de esta noción en el análisis de la individualidad biológica radica en el hecho de que una de las condiciones indispensables para que una entidad sea considerada como individuo es que esta entidad pueda tener una fitness propia. Esto la convierte en una entidad que puede ser seleccionada.
}

Revista de Humanidades de Valparaíso, 2019, No 14, 335-362 
supervivencia). ${ }^{18}$ La transferencia de fitness implica que los elementos o funciones que tienen un vínculo directo con la aptitud de los individuos, y por lo tanto tienen un vínculo directo con las funciones reproductivas y de supervivencia, deben "exportarse" al siguiente nivel. Esta "exportación" se entiende como la forma en que opera la conciliación entre dos niveles de organización. Para los estudios sobre transición evolutiva, la cooperación es fundamental justamente porque permite la exportación de la fitness desde nivel inferior en beneficio del nivel superior (Michod y Herron 2006). Es así que podemos unirnos a la idea que Michod propone a partir de una correlación negativa de fitness; para que un grupo pueda coordinarse como un agente evolutivo, la fitness de los individuos debe disminuir proporcionalmente al aumento de la fitness del grupo. Esto puede pensarse como una transferencia de funciones de un nivel al otro. En efecto, decíamos que, al concebir la acción simultánea de la selección natural a más de un nivel, suponemos de hecho una coexistencia de esos niveles, sin los cuales todos estos se verían perturbados y no se estabilizarían como grupo. Sin embargo, esta coexistencia puede justificarse como consecuencia de la transferencia de las funciones de fitness desde el nivel inferior al superior.

Los eventos de las transiciones mayores se interpretan, bajo la tesis de Michod, como transiciones de la individualidad, enfocándose en los mecanismos de transferencia de esta individualidad al nivel superior. En este caso, la transferencia de fitness permite conciliar un nivel de organización con otro, a través de una disminución en la aptitud individual de los miembros y un aumento en la aptitud global del grupo (Hanschen et al. 2015). La lógica de esta tesis se basa en el hecho de que, si uno piensa en funciones que pueden representar la aptitud (por ejemplo, la función reproductiva o las funciones de supervivencia somática), una transferencia de estas funciones a un contexto grupal podría entonces mover las fuerzas de selección individuales al grupo. Por un lado, porque los individuos que han transferido funciones que tienen un vínculo directo con la aptitud diferencial, ya no tendrán su independencia individual, por lo que su aptitud individual dependerá absolutamente de las relaciones que entrega en el grupo. Por otro lado, una transferencia de funciones al grupo permite que este grupo sea seleccionado como grupo, lo que define su estatuto de individuo susceptible de ser seleccionado.

Conjuntamente, la transferencia de funciones, que es el resultado de la especialización, es un buen ejemplo de la distribución de funciones en un dominio biológico más amplio. De hecho, la especialización de los individuos en elementos reproductivos y elementos somáticos puede interpretarse como una redistribución funcional, en el sentido de que las funciones de desarrollo, reproducción y supervivencia se redistribuyen a través del grupo y, más concretamente, en el organismo multicelular, a través de linajes celulares. Así,

\footnotetext{
${ }^{18}$ Esta diferencia entre viabilidad y fertilidad grafica la diferencia entre especialización y división del trabajo funcional fisiológico y reproductivo.
} 
division of labor among lower level units specialized in the basic fitness components of the group enhances the individuality of the group. Once cells are specialized in one of the necessary components of fitness, say reproduction or viability, they can no longer exist outside of the group and the fitness of the group is no longer the average fitness of the cells belonging to the group. (Hanschen et al. 2015, p. 167)

Por ejemplo, en el caso de los organismos sociales actuales, una vez que se lleva a cabo la división del trabajo entre los miembros, los miembros que corresponden al linaje somático ahora se ven obligados a cooperar para el correcto funcionamiento del conjunto. Así, la distribución funcional de los linajes celulares parece ser un buen ejemplo de dependencia constitutiva derivada de la especialización. Finalmente, la diferenciación funcional, como principio fundamental para establecer una dependencia fuerte entre los miembros de un dominio biológico discreto, también se encuentra en grupos de organismos formados por diferentes especies, como en muchos casos de mutualismo y simbiosis a lo largo de la historia evolutiva ${ }^{19}$. Esto corresponde bien al caso de las mitocondrias, que han perdido la función de replicación totalmente independiente, pero en su lugar ha asumido la función de proporcionar energía a partir de la producción de ATP en el ensamblaje celular ${ }^{20}$.

\section{Consideraciones finales}

Volvamos a una de las preguntas centrales de la armonía entre niveles: si suponemos que una correlación negativa entre la parte y el todo es indispensable para la conciliación entre dos niveles de organización, ¿qué interés tienen los individuos en delegar una parte de sus funciones mientras estas funciones los hacen libres y autónomos? Se supone, por supuesto, que la colaboración, que implica una pérdida de autonomía individual, implica ciertos beneficios para las entidades de una comunidad. Pero quizás también sea posible responder de manera amplia, suponiendo que cuando cada unidad subordina parte de su autonomía al mantenimiento de una organización general, también facilita su propia realización individual, ya que el conjunto de relaciones le proporciona funciones que luego no tiene que realizar él mismo. Esta última sugerencia asume un principio mereológico común: cuando el todo se vuelve más complejo, las partes se vuelven más simples (Varzi 2016). Esta lógica mereológica coincide con la idea de Michod, y que nosotros hemos expresado bajo una correlación negativa: "during a transition from a lower to a higher level, we expect the fitness of the higher level to increase and the fitness of the lower level to decrease" (Michod 2011, p.170). Ciertamente, una transferencia de funciones conduce a

\footnotetext{
${ }^{19}$ Otros modelos de transición los tratamos en Hernandez y Vecchi (2019).

${ }^{20}$ Se debe notar que la perdida de la autonomía reproductiva de la mitocondria es parcial. No obstante, si bien la mitocondria todavía se reproduce por fisión (como la mitocondria ancestral), necesita de los recursos de la célula huésped (en particular de los genes que codifican para las polimerasas que regulan la replicación del ADN y la transcripción). Por esta misma razón, la distinción entre la división del trabajo a través de la especialización fisiológica y reproductiva no es dicotómica.
}

Revista de Humanidades de Valparaíso, 2019, No 14, 335-362 
una simplificación de los individuos, pero al mismo tiempo permite la complejización del conjunto compuesto por individuos (véase nota 18). Si uno interpreta una transferencia de funciones como una especialización de las partes, la especialización de una parte es indudablemente más beneficiosa para el "todo", aunque la parte deba realizar acciones que no le sirvan directamente (por ej. sobre-producir un componente en particular o exponerse a peligros). A su vez, "el todo" trae cosas a la parte que ya no tiene que producir por sí sola (por ejemplo, el hecho de que las partes pueden verse favorecidas por un entorno más estable o que ciertos individuos puedan transmitir los genes de la comunidad).

Podemos tomar estas reflexiones para comprender un proceso de individualización colectivo: el fenómeno de la individualización colectiva pasa por un fenómeno de subordinación en el que las partes "delegan", hacia el nivel superior, funciones que las hacían autónomas. Hemos visto que este fenómeno puede interpretarse como un proceso de transferencia de funciones a un nivel superior, que se concreta, de acuerdo con los enfoques de las transiciones de la individualidad, mediante una transferencia de las funciones vitales. Esta transferencia de funciones restringe a los individuos a que pertenezcan a la nueva estructura para lograr su ontogénesis. Es por eso que una conciliación entre dos niveles pasa por una jerarquización que toma forma de constricción del todo hacia las partes. De ahora en adelante, interpretamos este fenómeno como una transferencia de autonomía.

También hemos visto que este proceso de transferencia de funciones se traduce concretamente en un fenómeno de especialización. Este fenómeno consiste en la especialización de individuos en elementos funcionales indispensables. El ejemplo de la multicelularidad nos proporciona una representación muy instructiva: la especialización funcional que destaca la multicelularidad consiste en separar las células dedicadas a la supervivencia y al mantenimiento del organismo, de las células dedicadas a la reproducción y la herencia ${ }^{21}$. De hecho, este fenómeno de división funcional hace posible comprender la estabilidad del grupo de individuos a partir de una interdependencia irreversible entre estos individuos: las unidades independientes que eran capaces de reproducirse y sobrevivir por si solas, sólo pueden hacerlo en un contexto relacional específico. En otras palabras, decimos que las unidades se han vuelto dependientes del nuevo dominio biológico del que ahora forman parte. La especialización de las funciones, establecida como una etapa crucial para que un conjunto de individuos se convierta en un solo individuo, se ha estudiado a través de la evolución de la cooperación, y esto presupone la aparición y la evolución de mecanismos que restringen la posibilidad de conflicto entre individuos en un grupo. Así, un grupo de individuos se convierte en un solo individuo estable, susceptible de sufrir los impactos de la selección natural a su nivel de organización.

\footnotetext{
${ }^{21}$ Es importante señalar que, justamente en este caso, se debe hablar más bien de transferencia parcial y no total (como sería el caso de transferencia de funciones de los insectos eusociales, en donde una casta se vuelve estéril y en donde la reproducción ocurre totalmente a nivel del grupo). En efecto, las células de la línea somática pueden seguir haciendo mitosis y reproduciéndose. Sin embargo, la especialización de línea germinal implica que ésta es la única capaz de reproducir al grupo.
}

Revista de Humanidades de Valparaíso, 2019, No 14, 335-362 
De lo anterior, podemos agregar lo siguiente:

En primer lugar, cuando tenemos un sistema en proceso de individualización, podemos establecer un criterio de robustez de la relación entre las partes a través de la irreversibilidad de sus asociaciones. En una organización donde los intercambios funcionales son más débiles, encontraremos relaciones que son menos dependientes: su nivel de acoplamiento será más reversible. Sin embargo, en los sistemas donde existe una irreversibilidad en sus relaciones, las partes tienen más dificultades para vincularse fuera de este dominio relacional específico, debido a la dependencia establecida con sus pares. Por lo tanto, creemos que las transiciones evolutivas corresponden a un caso de irreversibilidad funcional mayor, por el cual las partes han entablado relaciones constitutivas irrevocables.

En segundo lugar, nos gustaría asociar la noción de irreversibilidad con la noción de organismalidad (organismality) propuesta por Queller y Strassmann (Queller and Strassmann 2009). En efecto, los niveles de organización pueden establecerse por los grados de cooperación y ausencia de conflicto entre las partes, cuyos altos niveles de dependencia entre las partes demuestran una irreversibilidad de las relaciones. De esta manera, la organismalidad, como principio normativo que impone dos condiciones al organismo - alta colaboración y muy bajo nivel de conflicto-, nos permite pensar en grados de interacción $^{22}$ en el sentido de que las comunidades pueden tener diferentes niveles de conflicto, colaboración e irreversibilidad relacional. De esta manera, los altos niveles de colaboración y los bajos niveles de conflicto implican una irreversibilidad de los intercambios funcionales que mantienen los individuos, en los que los altos niveles de dependencia obligan a los individuos a colaborar en el mismo dominio relacional.

Tercero, nos gustaría recordar que los mecanismos que explican cómo se pueden controlar los conflictos involucran funciones colectivas. Todos los elementos que hemos considerado aquí (la división del trabajo, las separaciones funcionales en tipos de células, la interdependencia irreversible entre individuos, etc.) requieren la emergencia de funciones específicas de control de conflicto. Uno de los ejemplos representativos de la aparición de estos tipos particulares de funciones es algo que podemos llamar "constricciones del desarrollo". Este tipo de constricciones pueden haber surgido a partir de la interacción entre organismos a través de un proceso de agregación gradual. De hecho, aunque la evolución de la multicelularidad ha permitido la adaptación de la función del desarrollo hacia mecanismos precisos y complejos de fragmentación de una sola célula madre (división celular), no debemos olvidar que este fenómeno es el resultado inconfundible de un proceso histórico de agregación para lo cual todavía tenemos ejemplos vivos, como la familia de algas Volvox. Volvox es un alga que cubre un gran rango de complejidad organizacional. Encontramos algas de tipo unicelular y colonial, así como de tipo multicelular con una división del trabajo de tipo germen-soma. Estas algas son organismos modelo ideales para

\footnotetext{
${ }^{22}$ Esto implica que la organismalidad es una propiedad gradual, no categórica, del "todo" durante el proceso de individuación colectivo).
} 
abordar los problemas fundamentales relacionados con la transición a la multicelularidad y para construir principios teóricos que caracterizan esta transición (Hallmann 2011). Por ejemplo, Volvox carteri tiene dos tipos de células en su estado maduro; células reproductivas de tipo germinal, que no tienen senescencia (o muerte celular programada) conocida hasta la fecha, y células somáticas que corresponden a la motilidad de la colonia (Kirk 1988). El ejemplo de Volvox puede interpretarse como una transición evolutiva viva, y la variedad de comportamientos de este género de algas ha servido como modelo para comprender la transición mayor de la vida unicelular a la vida multicelular, así como la evolución de la multicelularidad y los mecanismos de diferenciación celular, lo que toca varias cuestiones fundamentales en la biología evolutiva (Herron 2016).

La importancia de este ejemplo es que resalta la necesidad de desarrollar funciones que controlen la agregación celular, a través de la división funcional, para lograr un ajuste comunitario. La agregación, un fenómeno que podemos claramente asociar con la naturaleza dinámica y social de la vida (y de su evolución química), es el sustrato fundamental del proceso de individualización colectiva. Por lo tanto, proponemos interpretar los procesos de desarrollo actual, incluidos los organismos sociales multicelulares, como un proceso de agregación o composición controlada. En otros términos, el fenómeno del desarrollo, como función emergente de una colectividad, no es más que un proceso de agregación bajo constricción; a saber, una optimización del fenómeno de agregación a partir de la autoproducción controlada de una gran parte del material biológico para organizar el organismo durante la embriogénesis. De hecho, todos los mecanismos de control que observamos actualmente (selección parental, proliferación unicelular, especialización, etc.) han sido optimizaciones de las agregaciones colectivas, cuyo ejemplo más representativo lo encontramos bajo la forma de constricciones del desarrollo.

Finalmente, es natural cuestionar también el motor final que impulsa a individuos a asociarse. Nuestro propósito es más humilde. Hemos querido mostrar simplemente las diversas facetas que incluyen el fenómeno de la asociación, dejando sus causas, por el momento, a los estudios de ecología evolutiva. Estos estudios abordan los fenómenos de conformación de nichos asociativos que responden a necesidades específicas: la ventaja que la asociación permite frente a los depredadores, el refugio o el control de un espacio ecológico, la resistencia a las condiciones adversas, la optimización del sistema o rendimiento en general, etc. Así, la explicación de las causas que llevan a los individuos a asociarse pertenece a los estudios y reflexiones más profundas sobre las "motivaciones", siempre en términos de selección y procesos no adaptativos, que encontramos detrás de estos organismos y comportamientos. En los casos de asociaciones sinérgicas, todas las variedades de posibles causas, ya sean contingentes o genéricas, explican la persistencia de tales asociaciones a lo largo del tiempo por el beneficio que implica la asociación. En este artículo, hemos mostrado cómo pueden tener lugar estas asociaciones, sin entrar en teorizaciones sobre las causas de la complejización de la naturaleza por parte de la asociación. 


\section{Agradecimientos}

Davide Vecchi agradece el suporte financiero de FCT - Fundação para a Ciência e a Tecnologia (DL57/2016/CP1479/CT0072; Grant N. UID/FIL/00678/2019; R\&D Project Grant PTDC/FER-HFC/30665/2017 "Emergence in the Natural Sciences: Towards a New Paradigm") y del Fondo Nacional de Desarrollo Científico y Tecnológico de Chile (Grant N. 1171017).

\section{Referencias bibliográficas}

Baron, C. (2013). The handicap principle and the argument of subversion from within. Studies in History and Philosophy of Biological and Biomedical Sciences, 44(3): 347-355.

Bedau, M. A. (2009). The Evolution of Complexity. En Anouk Barberousse, Michel Morange, Thomas Pradeau (eds.), Mapping the Future of Biology. Coll. Boston Studies in the Philosophy of Science, pp. 111-130. Dordrecht: Springer.

Buss, L. W. (1987). The Evolution of Individuality. Princeton, N.J.: Princeton University Press.

Calcott, B., Sterelny, K., Szathmáry, E. (eds.) (2011). The Major Transitions in Evolution Revisited. Coll. Vienna Series in Theoretical Biology. Cambridge, Mass.: MIT Press.

Clarke, E. (2010). The problem of biological individuality. Biological Theory, 5(4): 312-325.

Darwin, C. (2008). L'origine des espèces, Paris: Flammarion.

Doolittle, W. F., Bapteste, E. (2007). Pattern pluralism and the Tree of Life hypothesis. Proceedings of the National Academy of Sciences, 104(7): 2043-2049.

Dupré, J., O'Malley, M. A. (2009). Varieties of Living Things: Life at the Intersection of Lineage and Metabolism. Philosophy and Theory in Biology, 1(20170609).

Furusawa, C., Kaneko, K. (1998a). Emergence of multicellular organisms with dynamic differentiation and spatial pattern. Artificial Life, 4(1): 79-93.

Furusawa, C., Kaneko, K. (1998b). Emergence of rules in cell society: Differentiation, hierarchy, and stability. Bulletin of Mathematical Biology, 60(4): 659-687.

Furusawa, C., Kaneko, K. (2002). Origin of multicellular organisms as an inevitable consequence of dynamical systems. The Anatomical Record, 268(3): 327-342.

Ghiselin, M. T. (1971). The individual in the Darwinian revolution. New Literary History, 3(1): 113-134.

Godfrey-Smith, P. (2009). Darwinian populations and natural selection. Oxford: Oxford University Press.

Goodwin, B. C. (2001). How the Leopard Changed Its Spots: The Evolution of Complextiy. Princeton, NJ: Princeton University Press.

Hallman, A. (2011). Evolution of reproductive development in the volvocine algae. Sexual Plant Reproduction, 24(2): 97-112. doi: 10.1007/s00497-010-0158-4

Revista de Humanidades de Valparaíso, 2019, No 14, 335-362

(c) $(1)(9)$ CC BY-NC-ND 
Hanschen E., E. Shelton, D., Michod, R. (2015). Evolutionary Transitions in Individuality and Recent Models of Multicellularity. En Inaki Ruiz Trillo, Aurora Nedelcu (eds.), Evolutionary Transitions to Multicellular Life, pp. 165-188. London: Springer.

Hernandez, I., Vecchi, D. (2019). The Interactive Construction of Biological Individuality Through Biotic Entrenchment. Frontiers in Psychology 10:2578. doi: 10.3389/fpsyg.2019.02578

Herron, M. D. (2016). Origins of multicellular complexity: Volvox and the volvocine algae. Molecular Ecology, 25(6): 1213-1223.

Heylighen F. (1999). The Growth of Structural and Functional Complexity during Evolution. En F. Heylighen, J. Bollen, A. Riegler (eds.), The Evolution of Complexity, pp. 17-44. Dordrecht: Kluwer Academic.

Hull, D. L. (1980). Individuality and selection. Annual Review of Ecology and Systematics, 11(1): 311-332.

Hull, D. L. (1978). A Matter of Individuality. Philosophy of Science, 45(3): 335-360.

Huneman P. (2013). Adaptations in transitions: how to make sense of adaptation when beneficiaries emerge simultaneously with benefits? En Philippe Huneman, Frédéric Bouchard (eds.), From Groups to Individuals: Evolution and Emerging Individuality, pp. 141-172. Cambridge: MIT press.

Kaneko, K., Furusawa, C. (2000). Robust and irreversible development in cell society as a general consequence of intra-inter dynamics. Physica A: Statistical Mechanics and its Applications, 280(1-2): 22-33.

Kaneko, K., Yomo, T. (1999). Isologous diversification for robust development of cell society. Journal of Theoretical Biology, 199(3): 243-256.

Kirk, D. L. (1988). The ontogeny and phylogeny of cellular differentiation in Volvox. Trends in Genetics, 4(2): 32-36.

Leigh, G. (2010). The group selection controversy. Journal of Evolutionary Biology, 23(1): 6-19.

Leigh, E. G., Vermeij, G. J. (2002). Does natural selection organize ecosystems for the maintenance of high productivity and diversity? Philosophical Transactions of the Royal Society B: Biological Sciences, 357(1421): 709-718.

Lewontin, R. C. (1970). The units of selection. Annual Review of Ecology and Systematics, 1(1): $1-18$.

Margulis, L., Sagan, D. (2002). Acquiring Genomes: A Theory of the Origins of Species. New York, NY: Basic Books.

Martens, J. (2012). L'évolution des organisations biologiques : vers une théorie unifiée de la coopération et du conflit. Paris: Paris 1.

Maynard Smith, J., Szathmáry, E. (2010). The Major Transitions in Evolution. Oxford: Oxford University Press. 
Maynard Smith, J., Szathmáry, E. (2009). The Origins of Life: From the Birth of Life to the Origin of Language. Oxford: Oxford University Press.

Mayr E. (1997). The objects of selection. Proceedings of the National Academy of Sciences, 94(6): 2091-2094.

Mayr, E. (ed.) (1998). The Evolutionary Synthesis: Perspectives on the Unification of Biology. Cambridge, Mass.: Harvard University Press.

McShea, D. W. (2017). Perspective metazoan complexity and evolution: is there a trend? Evolution, 50(2): 477-492.

McShea, D. W. (2001). The hierarchical structure of organisms: a scale and documentation of a trend in the maximum. Paleobiology, 27(2): 405-423.

McShea, D. W., Brandon, R. N. (2010). Biology's First Law: The Tendency for Diversity and Complexity to Increase in Evolutionary Systems. Chicago, London: University of Chicago Press.

McShea, D. W., Hordijk, W. (2013). Complexity by subtraction. Evolutionary Biology, 40(4): 504-520.

Michod, R. E. (2011). Evolutionary Transitions in Individuality: Multicellularity and Sex. En Brett Calcott, Kim Sterelny (eds.), The Major Transitions in Evolution, pp. 169-198. Cambridge, Mass.: MIT Press.

Michod, R. E. (2007). Evolution of individuality during the transition from unicellular to multicellular life. Proceedings of the National Academy of Sciences, 104(1): 8613-8618.

Michod, R. E, Nedelcu, A. M. (2003). On the reorganization of fitness during evolutionary transitions in individuality. Integrative and Comparative Biology, 43(1): 64-73.

Michod, R. E, Roze, D. (2001). Cooperation and conflict in the evolution of multicellularity. Heredity, 86(1): 1-7.

Michod, R. E, Herron, M. (2006). Cooperation and conflict during evolutionary transitions in individuality. Journal of evolutionary biology, 19: 1406-1409; discussion 1426.

Newman, S. A., Bhat, R. (2009). Dynamical patterning modules: a "pattern language" for development and evolution of multicellular form. The International Journal of Developmental Biology, 53(5-6): 693-705.

Newman, S. A., Bonner, J. T. (2016). Multicellularity: Origins and Evolution. Cambridge, Mass.: MIT Press.

Newman, S. A., Forgacs, G., Muller, G. B. (2006). Before programs: the physical origination of multicellular forms. The International Journal of Developmental Biology, 50(2-3): 289-299.

Okasha, S. (2008). Evolution and the levels of selection. Oxford, New York: Clarendon Press, Oxford University Press. 
O’Malley, M. A., Wideman, J. G., Ruiz-Trillo, I. (2016). Losing Complexity: The Role of Simplification in Macroevolution. Trends in Ecology \& Evolution, 31(8): 608-621.

Pepper J. W., Herron. M. D. (2008). Does biology need an organism concept? Biological Reviews of the Cambridge Philosophical Society, 83(4): 621-627.

Pigliucci, M. (2010). Okasha's evolution and the levels of selection: toward a broader conception of theoretical biology. Biology \& Philosophy, 25(3): 405-415. doi: https://doi.org/10.1007/ s10539-010-9197-3

Queller, D. C. (2000). 13. Philosophical Transactions of the Royal Society of London B: Biological Sciences, 355(1403): 1647-1655.

Queller, D. C., Strassmann, J. E. (2009). Beyond society: the evolution of organismality. Philosophical Transactions of the Royal Society B: Biological Sciences, 364(1533): 3143-3155.

Ruiz-Mirazo, K., Moreno, A. (2012). Autonomy in evolution: from minimal to complex life. Synthese, 185(1): 21-52.

Sober, E., Wilson, D. S. (2003). Unto Others: The Evolution and Psychology of Unselfish Behavior. Cambridge, Mass.: Harvard University Press.

Solé, R. V., Goodwin, B. C. (2000). Signs of Life: How Complexity Pervades Biology. New York, NY: Basic Books.

Szathmáry, E. (2015). Toward major evolutionary transitions theory 2.0. Proceedings of the National Academy of Sciences, 112(33): 10104-10111.

Taylor, F. J. R. (1976). Autogenous theories for the origin of eukaryotes. Taxon, 25(4): 377.

Varzi, A. (2016). Mereology. En Edward N. Zalta (ed.), The Stanford Encyclopedia of Philosophy. Stanford: Metaphysics Research Lab, Stanford University. https://plato.stanford.edu/ entries/mereology/

Vecchi, D., Hernández, I. (2014). The epistemological resilience of the concept of morphogenetic field. En Alessandro Minelli y Thomas Pradeu (eds.), Towards a Theory of Development, pp. 79-94. Oxford: Oxford University Press.

West, S. A., Fisher, R. M., Gardner, A., Kiers E., T. (2015). Major evolutionary transitions in individuality. Proceedings of the National Academy of Sciences of the United States of America, vol. 112(33): 10112-10119.

Wilson, D. S., Sober, E. (1989). Reviving the superorganism. Journal of theoretical Biology, 136(3): 337-356.

Wilson, D. S. (1997). Altruism and Organism: Disentangling the Themes of Multilevel Selection Theory. The American Naturalist, 150(S1): S122-S134.

Wilson, R. A. (2005). Genes and the Agents of Life: The Individual in the Fragile Sciences Biology. Cambridge: Cambridge University Press. 
Individuación colectiva y emergencia de la organismalidad

Isaac Hernández; Davide Vecchi

Wolf, Y. I., Koonin, E. V. (2013). Genome reduction as the dominant mode of evolution. BioEssays: News and Reviews in Molecular, Cellular and Developmental Biology, 35(9): 829-837.

Revista de Humanidades de Valparaíso, 2019, No 14, 335-362

(c) $(\Theta \Theta C C$ BY-NC-ND 